\title{
Testing numerical implementations of strong-field electrodynamics
}

\author{
C. N. Harvey, ${ }^{1,2, *}$ A. Ilderton,,$^{2, \dagger}$ and B. King ${ }^{3, \ddagger}$ \\ ${ }^{1}$ Centre for Plasma Physics, Department of Physics and Astronomy, Queen's University Belfast, Belfast BT7 1NN, United Kingdom \\ ${ }^{2}$ Department of Applied Physics, Chalmers University of Technology, SE-41296 Gothenburg, Sweden \\ ${ }^{3}$ School of Computing and Mathematics, Plymouth University, Plymouth PL4 8AA, United Kingdom
}

(Received 26 September 2014; published 13 January 2015)

\begin{abstract}
We test current numerical implementations of laser-matter interactions by comparison with exact analytical results. Focusing on photon emission processes, it is found that the numerics accurately reproduce analytical emission spectra in all considered regimes, except for the harmonic structures often singled out as the most significant high-intensity (multiphoton) effects. We find that this discrepancy originates in the use of the locally constant field approximation.
\end{abstract}

DOI: 10.1103/PhysRevA.91.013822

PACS number(s): 12.20.Ds, 41.60.-m, 52.38.Ph

\section{INTRODUCTION}

The study of classical and quantum electrodynamical (QED) processes in strong background fields is currently a highly active research area, as advances in technology now allow fundamental physics to be tested using intense laser light [1-7]. The basic QED processes in strong backgrounds were thoroughly investigated soon after the invention of the laser itself [8,9], using simple laser field models. However, there is a limit to the field complexity and the number of reacting particles which can be considered before analytical calculations become too unwieldy. Numerical investigation offers one route out of this impasse.

An increasingly common numerical model of QED in intense fields is based on particle-in-cell (PIC) codes. There are a number of such codes in use [10-12], and while they differ in the details, they share a common implementation of lasermatter interactions; particles in an intense field are propagated classically thorough discrete time steps, and statistical event generators are used to determine, at each step, the likelihood and result of various QED processes [13-17] (for reviews see Refs. [18,19]).

In this article we attempt to assess the accuracy of these numerical techniques in the high-intensity regime appropriate to modern laser systems which reach, and will soon exceed [20-22], focal intensities of $10^{22} \mathrm{~W} / \mathrm{cm}^{2}$ [23]. For some previous comparisons of theory and simulation, see Refs. [18,24].

While existing analytical results are largely confined to scattering probabilities (i.e., asymptotic results) in simple field configurations and for small numbers of initial and final state particles, the aim of numerical codes is to simulate, in real time, many-particle interactions in focused, structured laser pulses. Despite these differences, we show that it is indeed possible to make a direct comparison between numerical predictions and exact analytical results, and thereby test the assumptions that go into the numerical model. Our interest here is not in providing phenomenological results for comparison with experiment, but in addressing the question of

\footnotetext{
*christopher.harvey@ chalmers.se

†anton.ilderton@chalmers.se

†b.king@plymouth.ac.uk
}

whether the currently employed statistical modifications to PIC simulations can reproduce well-known and well-understood intensity effects in QED. We can therefore employ a simple beam model in order to have a reliable analytical prediction. Furthermore, we restrict our attention to multiple-photon emissions from an electron in an intense field and consider the best-known signatures of intensity and multiphoton effects from the literature.

The article is organised as follows. We begin in Sec. II by briefly reviewing the main features of the photon emission spectrum in nonlinear Compton scattering. There follows a review of commonly used numerical schemes. In Sec. III we describe the observables of interest and compare the analytical and numerical calculations of these for a variety of laser and electron parameters. An analysis of some differences is given in Sec. IV and we conclude in Sec. V.

\section{APPROACHES TO STRONG-FIELD QED}

We consider an electron moving in an electromagnetic field, and its emission of photons. We model the background as a plane wave, a decision motivated not by phenomenological interest but rather by a desire to perform as rigorous a comparison as possible; everything we need can be calculated exactly in plane waves.

The restriction to photon emission implies neglecting, e.g., pair production from emitted photons [25-29] or pair production via trident [30-34]. Here it is useful to recall the two standard parameters important in this analysis, $a_{0}=e E / m \omega$ (for electron mass and charge $m$ and $e$, external field strength and frequency $E$ and $\omega$, where $\hbar=c=1$ ), which quantifies the classical nonlinearity, and $\chi_{e}=e|F \cdot p| / m^{3} \sim \gamma E / E_{\mathrm{cr}}$, where $\gamma$ is the electron's gamma factor and $E_{\mathrm{cr}}=m^{2} / e[35-$ 37], which quantifies the quantum nonlinearity [38]. For $\chi_{e} \gtrsim$ 1 quantum effects generally become probable and photonseeded pair production is more likely to occur. One finds (using the constant crossed field model) that electron-seeded pair creation via a virtual photon can be neglected when $a_{0} \gg 1[31,34]$, as can secondary photon emission via a virtual electron [39]. Further, radiation reaction effects become important only when $\alpha a_{0} \chi_{e} N_{c} \approx 1$, where $N_{c}$ is the number of cycles in the field $[7,40]$.

Thus, in order to study photon emissions without additional effects, one should be in a regime where $\chi_{e}$ is less than unity. 
We require however $a_{0} \gg 1$ so that we have strong nonlinear effects, and $\gamma$ may still be large, provided $\chi_{e} \lesssim 1$. We therefore restrict ourselves to this regime. We stress though that our analysis can be extended, as will become clear, both into the quantum regime and to, e.g., stimulated pair production, which exhibits an emission spectrum similar to that of photon emission [25-29].

\section{A. Nonlinear Compton scattering}

With "nonlinear Compton scattering," we refer to the emission of a photon from an electron in a plane wave background. For simplicity of calculation and presentation we consider a circularly polarized, monochromatic plane wave traveling in the positive $z$-direction. The electromagnetic fields are therefore composed of photons of four-momentum $k_{\mu}$, where $k \cdot x=\omega(t-z)$, and take the form, writing $\phi=k \cdot x$ from here on,

$$
\begin{aligned}
& \mathbf{E}_{\text {mono. }}(x)=E(\cos \phi, \sin \phi, 0), \\
& \mathbf{B}_{\text {mono. }}(x)=E(-\sin \phi, \cos \phi, 0) .
\end{aligned}
$$

The intensity parameter $a_{0}$ characterizes the strength of interaction between the wave and the electrons.

Consider an electron with initial momentum $p_{\mu}$ and final momentum $p_{\mu}^{\prime}$ after emitting a photon of momentum $k_{\mu}^{\prime}$. The probability of emission $\mathbb{P}$ is calculated as usual from the appropriate $S$-matrix element. Due to the periodicity and infinite duration of the wave, this probability is infinite. However, dividing out this infinite factor, the emission rate $W$ per unit $\phi$ (lightfront time) is finite and can be written as a sum over partial rates $W_{n}[8,9]$,

$$
W=\sum_{n=1}^{\infty} W_{n}
$$

where $W_{n}$ describes emissions in a finite kinematic range that correspond to a "harmonic." (Comprehensive discussions can be found in any of Refs. [3,7-9,41-44]; we review here only the relevant details.) The total and partial rates can be expressed as integrals over differential rates in the outgoing photon frequency and momentum. Although it is common to plot the spectrum as a function of frequency, a variable arising naturally in the QED calculation is the "lightcone momentum fraction" $x$,

$$
x=\frac{k \cdot k^{\prime}}{k \cdot p^{\prime}}=\frac{k \cdot k^{\prime}}{k \cdot p-k \cdot k^{\prime}} .
$$

Defining the effective mass squared $m_{*}^{2}=m^{2}\left(1+a_{0}^{2}\right)[45,46]$, it can be shown that the kinematically allowed range of the $n$th harmonic is (see, e.g., Ref. [41])

$$
0 \leqslant x \leqslant y_{n}, \quad y_{n} \equiv n \frac{2 k \cdot p}{m_{*}^{2}} .
$$

The $n$th harmonic rate $W_{n}$ vanishes outside of this range. The harmonic decomposition (2) is due to the periodicity of the beam, but in fact any stretch of uniformly periodic field leads to such effects and provides the experimental signature of, e.g., the intensity-dependent effective mass [47]. Further, harmonic generation has been searched for and observed in several experiments [48-50].
Note that a given frequency is not necessarily found in a single harmonic range, as these can overlap or be disjoint in frequency space depending on initial conditions and harmonic number [41]. The allowed scattered photon frequencies $\omega_{n}^{\prime}$ in the $n$th harmonic obey, assuming a head-on collision between the electron and laser to illustrate,

$$
\omega_{n}^{\prime}=\frac{n \omega}{1+j_{n}(1-\cos \theta)},
$$

where $\theta$ is the photon scattering angle relative to the electron direction and

$$
j_{n}=\frac{n \omega / m-\gamma \beta+a_{0}^{2} \gamma(1-\beta) / 2}{\gamma(1+\beta)} .
$$

The behavior of $j_{n}$ characterizes much of the spectrum. When $j_{n}<0\left(j_{n}>0\right)$ the emission frequency is maximal for backscattering, $\theta=\pi$ (forward scattering, $\theta=0$ ). From Eq. (5) we see that $\omega_{n}^{\prime}(\theta=0)=n \omega$, so that for $j_{n}<0$ the scattered frequencies $\omega_{n}^{\prime}$ are blue-shifted relative to $n \times$ the laser frequency, and if $j_{n}>0$ they are red-shifted.

The emission spectrum acquires some particularly distinctive features for initial conditions such that $j_{n}=0$ for some $n$. In that case $\omega_{n}^{\prime}$ loses its $\theta$ dependence and the $n$th harmonic range collapses to a single point $\omega_{n}^{\prime}=n \omega$. Neighboring harmonics also collapse to very narrow peaks, resulting in a line-spectrum region (a comblike structure) within the full spectrum. Setting $j_{n}=0$ in order for the $n$th harmonic to collapse, we find that $a_{0}$ and $\gamma$ must be related by

$$
a_{0, \mathrm{crit}}^{2}(n) \equiv \frac{2(\gamma \beta-n \omega / m)}{\gamma(1-\beta)} .
$$

The behavior of $j_{n}$ gives us a useful separation of the system into three parameter regimes: "subcritical," $a_{0}<a_{0 \text {, crit }}$; "supercritical," $a_{0}>a_{0, \text { crit }}$; and "critical," $a_{0} \approx a_{0 \text {, crit. These }}$ three cases are examined numerically below.

\section{B. The numerical approach}

Two limitations on what can be achieved using exact analytical methods are as follows. The first is that the external fields in which scattering amplitudes can currently be calculated exactly do not include the spatial focusing of laser fields employed in experiments. The second limitation is that the complexity of $S$-matrix elements grows rapidly with the number of initial and final particles. A complete analytical description of a cascade [13-17], for example, is extremely challenging. We therefore turn now to the numerical models which offer a route to potentially overcome these limitations. We outline the shared general principles of currently employed codes, reviewed in Refs. [18,19], beginning with the use of the locally constant field (LCF) approximation.

In the high-intensity limit $a_{0} \gg 1$ the size of the radiation formation region is of the order $\lambda / a_{0} \ll \lambda$, where $\lambda=2 \pi / \omega$ is the laser wavelength [38]. Thus the laser field varies on a scale much larger than the formation region and so can be approximated as locally constant and crossed [8], allowing us to determine the probability of photon emission using the expression for the constant crossed field rate $\Gamma$ per unit 
time,

$$
\frac{d \Gamma}{d \chi_{\gamma}}=\frac{\alpha m}{\sqrt{3} \pi \gamma \chi_{e}}\left[\left(2+\frac{x^{2}}{1+x}\right) K_{2 / 3}(\tilde{\chi})-\int_{\tilde{\chi}}^{\infty} d y K_{1 / 3}(y)\right],
$$

where $K_{v}$ is the modified Bessel function, $\chi_{\gamma}=e\left|F \cdot k^{\prime}\right| / m^{3}$ for the emitted photon with momentum $k_{\mu}^{\prime}$, note that $x=$ $\chi_{\gamma} /\left(\chi_{e}-\chi_{\gamma}\right)$, and $\tilde{\chi}=2 x /\left(3 \chi_{e}\right)$. Although $d \Gamma / d \chi_{\gamma}$ diverges at small $\chi_{\gamma}$, the total rate of photon emission $\Gamma$, given by integrating Eq. (8) over all $\chi_{\gamma} \in\left[0, \chi_{e}\right]$, is finite. (This apparent softening of the usual infrared divergence in QED is explained in Ref. [51].) The rate (8) incorporates an averaging over electron spin and photon polarization as typical numerical codes do not resolve these degrees of freedom. As such, we compare numerical results with the appropriate spin and polarization-averaged analytic results. (Note that polarization could be included in the codes using, e.g., the results of Ref. [24].)

In numerical simulations, the electron is evolved along a classical trajectory over discrete time steps. After each step $\Delta t$ the following statistical routine is used to calculate the probability of photon emission and to correct the electron's momentum. A uniform random number $r \in[0,1]$ is generated and emission is deemed to occur if the condition $r \leqslant \Gamma \Delta t$ is satisfied, under the requirement $\Gamma \Delta t \ll 1$. Note that $d \Gamma / d \chi_{\gamma}$ (and $\Gamma$ ) are time-dependent quantities in the simulation, due to the temporal variation of the laser pulse and electron motion. Given that an emission event occurs, a second uniform random number $\zeta \in[0,1]$ is generated and the photon's $\chi_{\gamma}$ (and therefore its frequency) is determined as the root of the sampling equation ${ }^{1}$

$$
\zeta=\Gamma(t)^{-1} \int_{0}^{\chi_{\gamma}} d \chi_{\gamma} \frac{d \Gamma(t)}{d \chi_{\gamma}} .
$$

The photon momentum is then determined by $\chi_{\gamma}$ together with the assumption that the electron emits in the forward direction at high $\gamma$. In reality the emissions will be concentrated in a cone of opening angle $\gamma^{-1}$ [41,53]. Finally, the emitted photon momentum is subtracted from the electron momentum, i.e., the electron is recoiled, imposing the conservation law $\chi_{e} \rightarrow \chi_{e}-\chi_{\gamma}$ [38], and the simulation proceeds by propagating the electron (via the Lorentz equation) and the photon (on a linear trajectory) to the next time step. In this way, multiple emissions are described as sequential single-photon emissions, as in Eq. (8), occurring at discrete time intervals. Analytical discussions of double-photon emission are given in Refs. [54,55].

\section{COMPARISON OF ANALYTICAL AND NUMERICAL RESULTS}

The numerical model described above is not equivalent to the calculation of transition amplitudes in QED. In QED,

\footnotetext{
${ }^{1}$ In practice an infrared cutoff is used; i.e., the integral is performed from a lower limit $\chi_{\gamma} \sim 10^{-5}$, rather than zero, so that the codes do not include the emission of large numbers of low energy photons, which does not appreciably affect the electron's dynamics [18,52]. For an alternative event generator, see, e.g., Ref. [18].
}

scattering amplitudes are determined using asymptotic in and out states. No assumptions are made about the electron's trajectory in the laser. In the numerical method, on the other hand, we constantly track the electron's trajectory, and asymptotic scattering probabilities are combined with statistical routines to determine the likelihood of local transitions. As such the codes work at the level of the probability, or cross section, rather than at the amplitude level. This suggests that interference effects, which are an essential part of quantum mechanics, may not be properly included in the codes. (Indeed, this is why including higher-order processes such as trident is conceptually difficult.) In short, it should be checked whether the numerical model agrees with theory.

We calculate and compare analytical and numerical predictions for the observable $N_{\gamma}$, the average number of emitted photons. This is clearly something which can be easily extracted from the numerics; we run the code many times, always with the same initial conditions, and count the number of photons emitted into a particular (binned) angle with a particular (binned) frequency.

It may not be immediately obvious how to perform the analytical calculation, as we have described only the single-photon emission probability and have already mentioned the difficulty with going to higher orders. Fortunately, in the regime in which we are working, knowing the one-photon emission probability is enough because of the well-known infrared (IR) properties of electrodynamics [56-59]; see Ref. [60, §6] for an introduction.

The "probability" $\mathbb{P}$ can only be interpreted as such when it is much less than unity, due to neglected higher-order corrections from multiple soft photon emissions. ${ }^{2}$ It is more properly interpreted as the expected number of emitted photons $N_{\gamma}$, which is why it easily exceeds unity [60]. Similarly, the differential probability gives the differential number of produced photons, so

$$
\mathbb{P} \rightarrow N_{\gamma}=\int d \omega d \Omega \frac{d^{2} N_{\gamma}}{d \omega d \Omega} .
$$

This allows us to consider multiple emissions using the well-understood probability of single emission. This, and the identification (10), holds for low energies, as can be confirmed by calculating $N_{\gamma}$ classically and comparing with the classical limit of the QED emission probability, as in Ref. [63].

For the purposes of this study we use the single-particle QED code SIMLA, which works in the typical manner [64,65]. While a monochromatic wave gives the easiest analytical calculation of the emission spectrum, it is more difficult to work with numerically since we cannot run the simulation for an infinite period of time. However, the spectrum will be very similar to that in a plane wave with a long super-Gaussian time envelope, as demonstrated in Ref. [47]. For our numerical simulations we therefore take a circularly polarized plane wave with a 100-fs degree- 8 super-Gaussian time envelope,

$$
\mathbf{E}_{\text {super }}(x)=e^{-\left(\frac{2 \phi}{d}\right)^{8} \ln 2} \mathbf{E}_{\text {mono. }}(x),
$$

in which $d=100$ fs is the full width at half maximum and this is practically equal to the full duration of the pulse,

\footnotetext{
${ }^{2}$ IR effects in single- and multiple-photon emissions in background fields are studied in Refs. [51,61,62].
} 


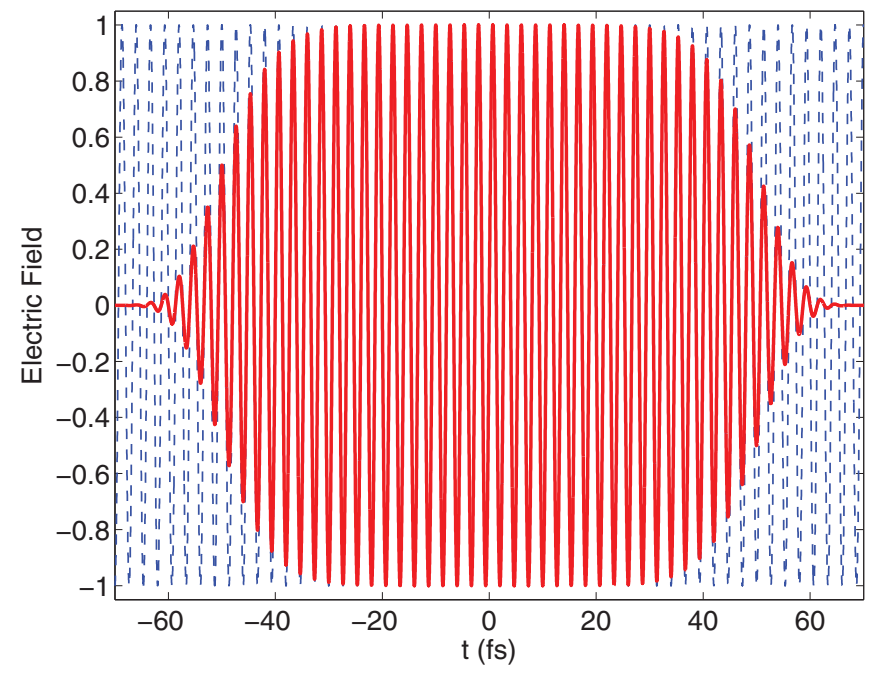

FIG. 1. (Color online) Comparison of the electric field strength (normalized to peak field strength) in one of the polarization directions for an infinite plane wave (dashed blue line) and a degree- 8 superGaussian wave of $100 \mathrm{fs}$ in duration (solid red line) $(\lambda=0.8 \mu \mathrm{m})$.

i.e., the pulse has almost a flat top, as shown in Fig. 1. We now investigate three parameter regimes and present illustrative comparisons between theory and numerics. (In the following plots the analytical amplitudes are normalized using the numerical results.)

\section{A. Supercritical}

We begin with a supercritical setup, $a_{0}>a_{0 \text {,crit }}(1)$ (which gives $\left.a_{0}>a_{0, \text { crit }}(n) \forall n \in \mathbb{N}^{+}\right)$. Specifically, we choose the parameters $a_{0}=80$ and $\gamma=10$ such that $a_{0}>a_{0 \text {, crit }}(1) \approx 20$. The analytical and numerical spectra are plotted in Figs. 2 and 3. It can be seen in Fig. 2 that the frequency spectra calculated using the two methods agree extremely well. Both

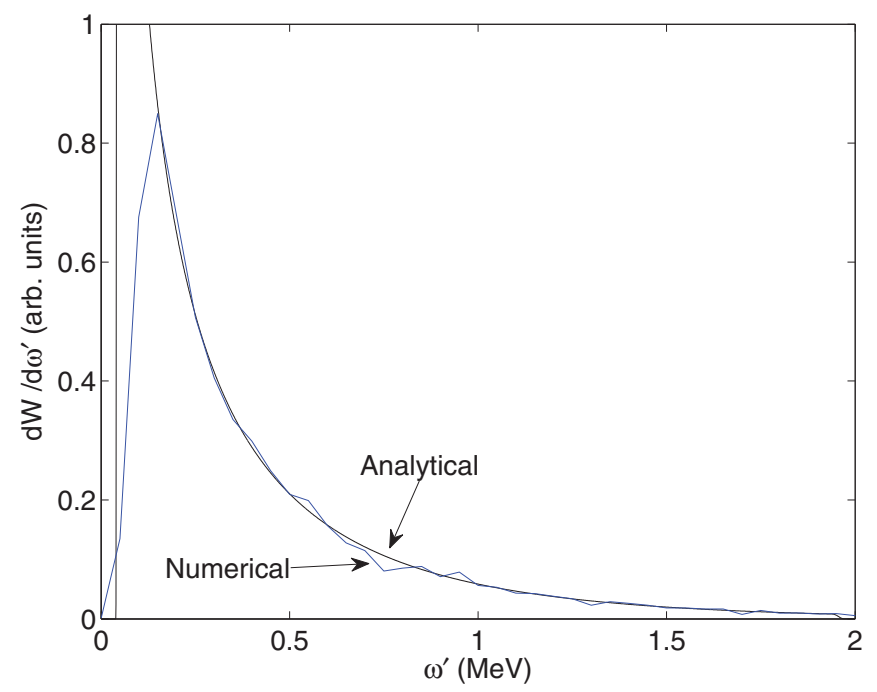

FIG. 2. (Color online) Comparison of analytical and numerical frequency emission spectra in the supercritical regime. The parameters are $a_{0}=80$ and $\gamma=10$. The black line shows the analytical calculation, and the blue (gray) line shows the statistical photon distribution from several thousand numerical runs.

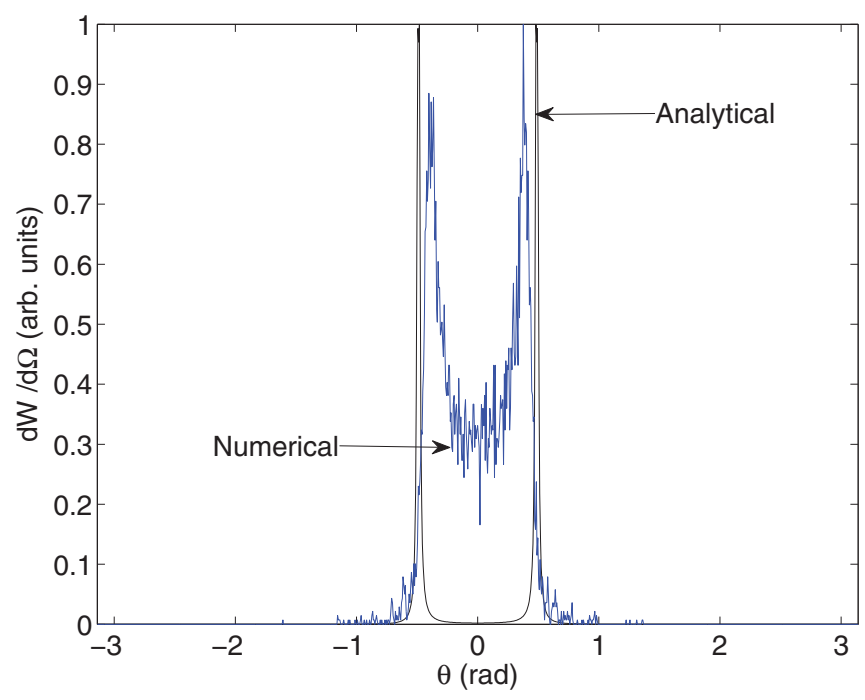

FIG. 3. (Color online) Comparison of analytical and numerical angular emission spectra in the supercritical regime. The parameters are $a_{0}=80$ and $\gamma=10$. The black line shows the analytical calculation, and the blue (gray) line shows the statistical photon distribution from several thousand numerical runs.

have the same structures and shape and both decay at the same rate. The only difference is that the numerical spectra falls off for low $\omega^{\prime}$, but this is simply because of the IR cutoff in the code and should not worry us. Note that the low-energy cutoff is not sharp because it is defined in terms of a minimum $\chi_{\gamma}$ rather than frequency. ${ }^{3}$

The angular emission rates are plotted in Fig. 3 where it can be seen that there is also fairly good agreement. The peaks for the numerical and analytical cases are in approximately the same locations and the rates of falloff for angles larger than the peak value are almost identical. Nevertheless the two spectra disagree for small angles. The reason for this is likely to be due to the fact that the code assumes that photons are emitted in the direction of motion of the electron, whereas in reality they would be emitted in a cone of opening angle $1 / \gamma \sim 0.1 \mathrm{rad}$.

\section{B. Subcritical}

The next setup we consider is the subcritical regime in which much of the clearly identifiable structure in the emission spectrum is found in the low-lying harmonics [41]. These, being characterized by frequencies equal to multiples of the laser frequency [with an intensity-dependent red-shift, see Eq. (5)] are often located below the infrared cutoff imposed in the codes. In order to lift these into the part of the spectrum resolved by the code we choose $\gamma=9000$ and again take $a_{0}=20$ ( $\chi_{e} \simeq 0.4$ ). For these parameters all harmonics up to $n \approx 10^{9}$ are blue-shifted; due to the falloff of the spectrum we can effectively say this applies to all harmonics. The frequency spectra are plotted in Fig. 4 and the angular rates in Fig. 5. In both cases there is, in general, a very good agreement between

\footnotetext{
${ }^{3}$ Additionally, there will be some noise at the low-frequency end of the spectrum from when the electron is in the lower intensity rise and fall of the super-Gaussian field.
} 


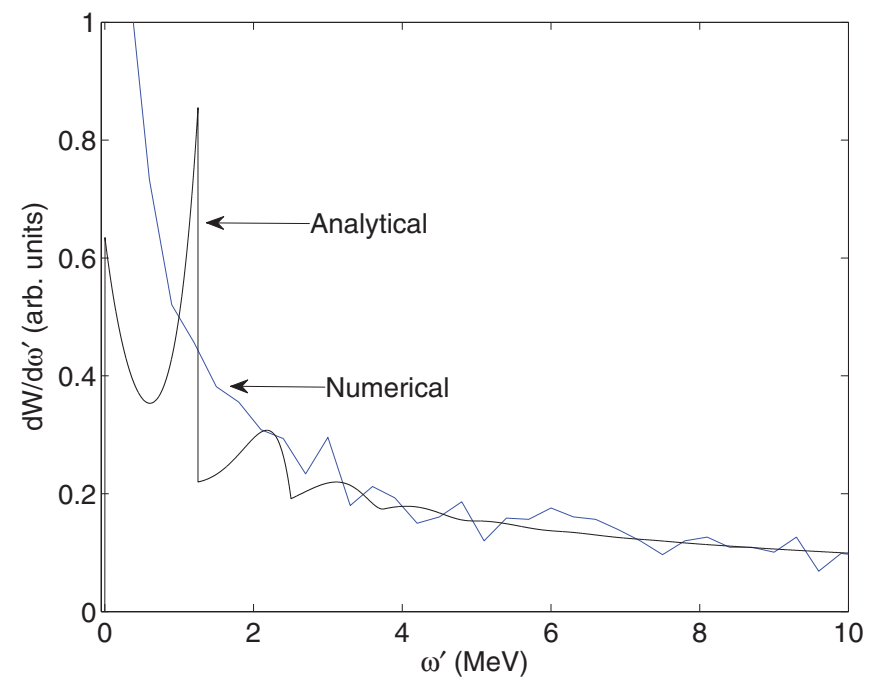

FIG. 4. (Color online) Comparison of the analytical (black) and numerical (blue or gray) frequency spectra for the case of subcritical $a_{0}$. The parameters are $a_{0}=20$ and $\gamma=9000$.

the numerical and analytical results. However we see in Fig. 4 that the structures of individual harmonics, which in this case sit at the lower frequency end of the spectrum, are missed by the code; instead there appears to be a smooth interpolation through them.

\section{Critical}

In the previous example we chose parameters to blue-shift the low-lying harmonics to high frequency. This required very high energy electrons, but even for moderate (readily attainable) energies and laser intensities the emission spectrum can naturally feature interesting structures which should be reproduced by the codes, as they fall into resolved energy regimes. One such feature is described by a collision at critical parameters in which a harmonic in the middle of the spectrum collapses.

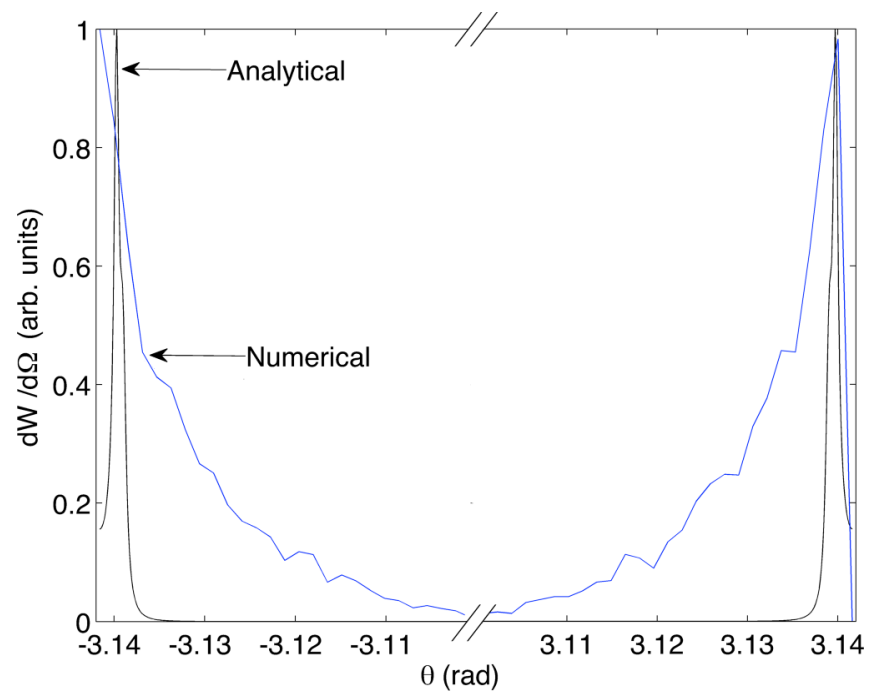

FIG. 5. (Color online) Comparison of the analytical (black) and numerical (blue or gray) angular emission rates for the case of subcritical $a_{0}$. The parameters are $a_{0}=20$ and $\gamma=9000$.

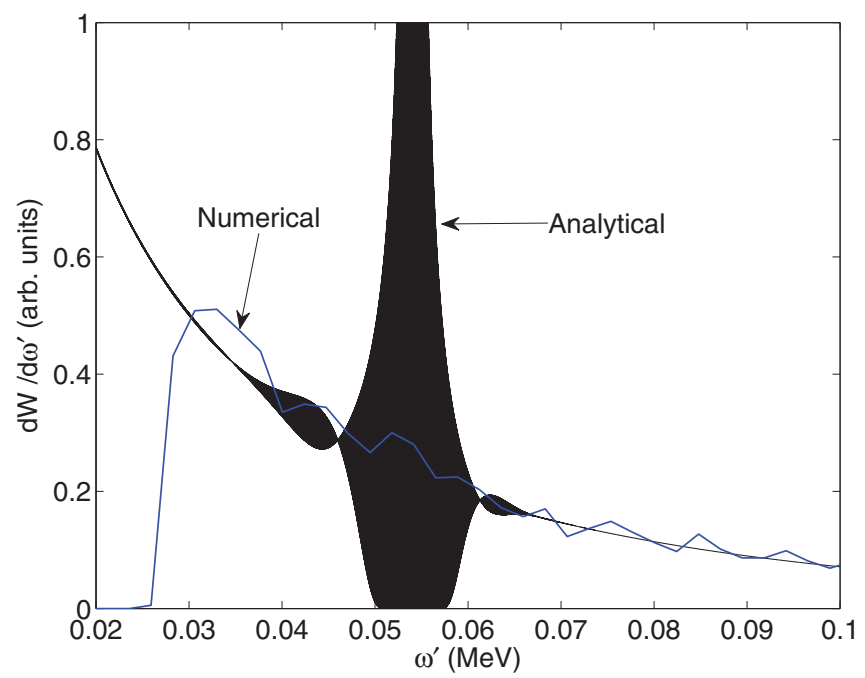

FIG. 6. (Color online) Comparison of the analytical (black) and numerical (blue or gray) frequency spectra for the case of critical $a_{0}$. The parameters are $a_{0}=30$ and $\gamma=15.078$.

We set the laser intensity to be $a_{0}=30$ and take $\gamma=$ 15.078. (There is a degree of fine-tuning here.) From Eq. (7) we see that this corresponds to a collapse of the particular harmonic $n=35000$ at $\omega^{\prime}=0.05415 \mathrm{MeV}$. The value of $n$ is irrelevant, what is important is the form of the spectrum; a harmonic collapses to a peak at a single point in the high-energy part of the spectrum, and neighboring harmonics collapse to very narrow lines, resulting in a region of the spectrum resembling a $\delta$ comb. Such combs occur for relatively long driving pulses and correspond to multiphoton peaks; these are seen in both photon emission [41,44] and in pair creation [28]. Compare and contrast with the coherent combs recently studied in Ref. [66] for photon emission.

The resulting emission spectra are plotted in Figs. 6-8. In Fig. 6 the peaked and rapidly oscillating comblike structure can be very clearly seen. Away from this part of the spectrum,

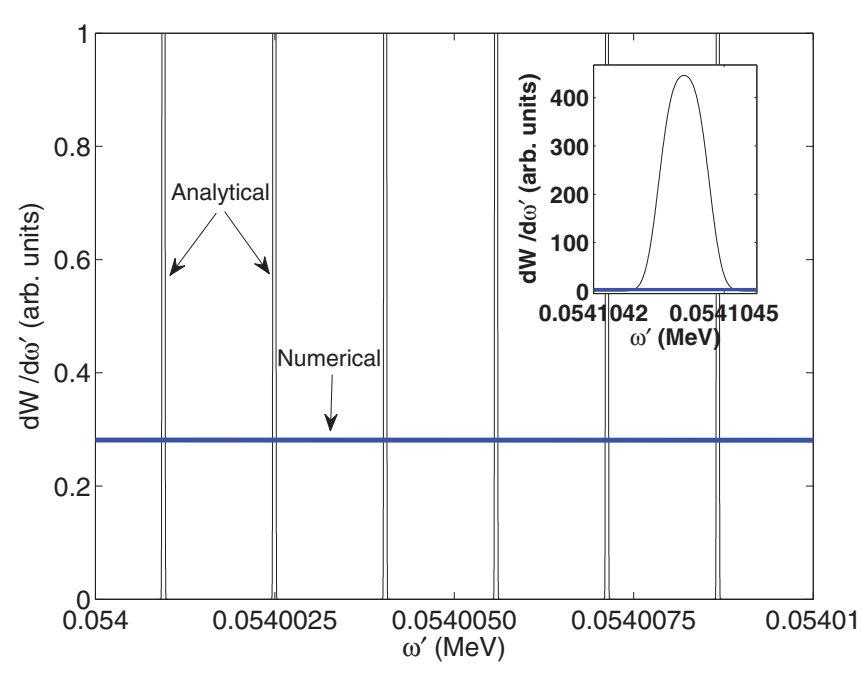

FIG. 7. (Color online) A zoom-in of the collapsed part of the spectrum in Fig. $6\left(a_{0}=30, \gamma=15.078\right)$. The inset shows a further zoom into an individual harmonic. 


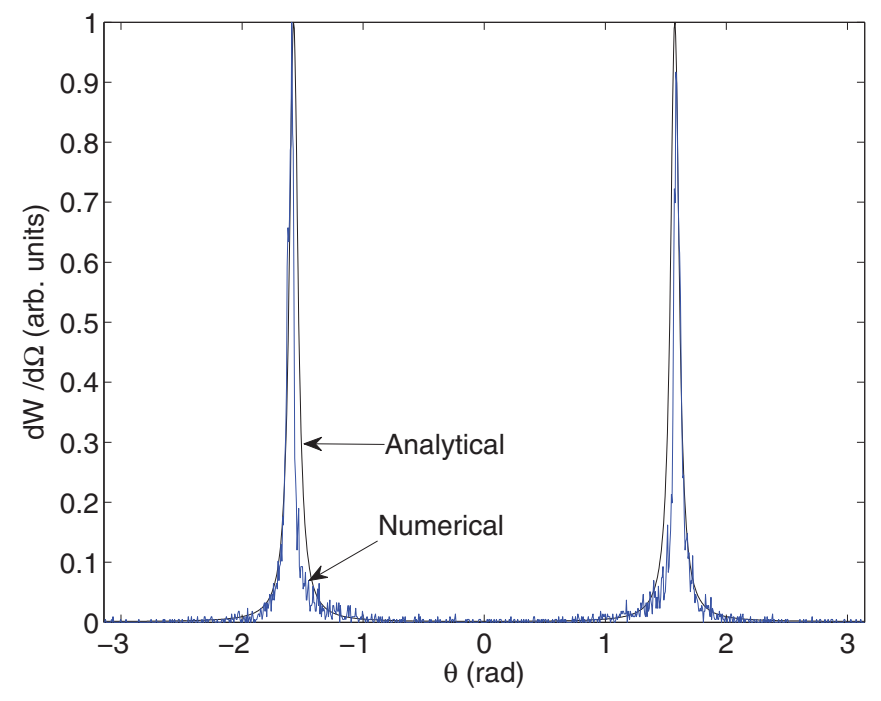

FIG. 8. (Color online) Comparison of the analytical (black) and numerical (blue/gray) angular emission rates for the case of critical $a_{0}$. The parameters are $a_{0}=30, \gamma=15.078$.

the analytical and numerical results agree very well once again. In the region of the comb structure however, and as can be seen clearly in the zoomed-in plot in Fig. 7, the numerical spectrum again does not resolve the harmonic peaks in the analytical spectrum; instead it appears to average over them. As can be seen in Fig. 8, though, the analytical and numerical angular rates agree extremely well.

It is instructive to also consider parameters that are slightly "off critical" in order to demonstrate how sensitive the spectrum is to initial conditions. We take $a_{0}=30$ and $\gamma=16$, so that the comblike part of the spectrum is shifted just out of the region we are considering. The frequency spectrum is shown in Fig. 9. Here the individual harmonics join together to produce a continuous spectrum, and we can see that the numerical results once again agree extremely well with the

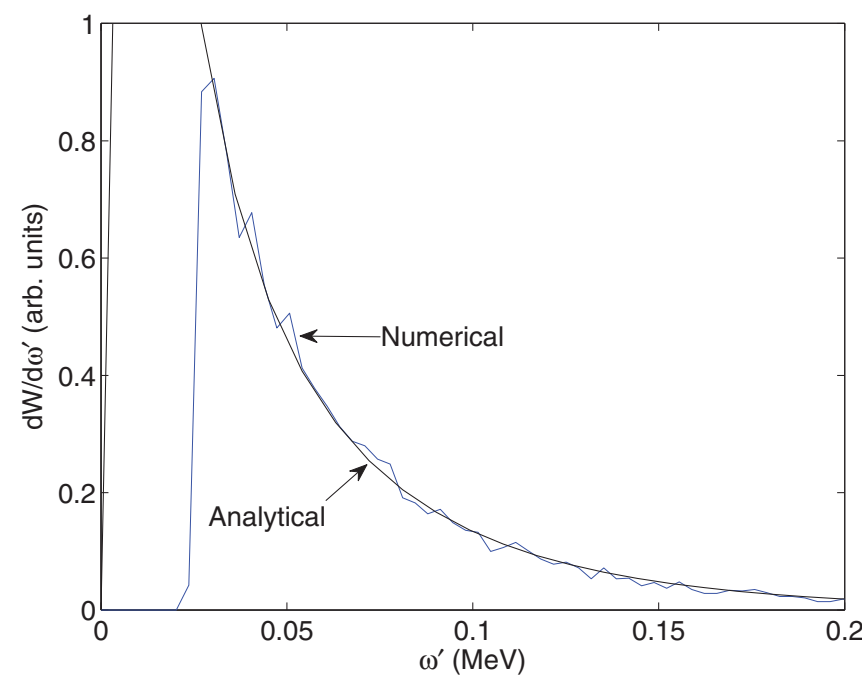

FIG. 9. (Color online) Comparison of the analytical (black) and numerical (blue or gray) angular emission rates for the case of nearcritical $a_{0}$. The parameters are $a_{0}=30$ and $\gamma=16$. analytical calculation. (To shift the critical harmonic beyond $0.2 \mathrm{MeV}$ in Fig. 9 one needs to increase $\gamma$ to 15.123. The spectral range in a plane wave is infinite, but the spectrum decreases exponentially at high frequency.)

\section{ANALYSIS}

Although the numerical model captures the coarse details of the emission spectra considered, it fails to reproduce any of the harmonic signatures that characterize emission in monochromatic waves. We have found that this discrepancy is best explained in terms of the LCF approximation. In accordance with earlier discussions we analyze this in the classical limit.

While the extension to quantum expressions is apparent, we postpone a detailed analysis to future work.

The total number of emitted photons, $N_{\gamma}$, can be calculated either from standard expressions $[53, \S 14]$ or from the classical limit of the nonlinear Compton scattering probability calculated in QED, as in Ref. [63]. Since $N_{\gamma}$ is given by an integral over the mod-square electromagnetic current generated by the electron [53], it can be written as a double integral over the external-field phase $\phi$,

$$
N_{\gamma}=\int d \phi d \phi^{\prime} \frac{d^{2} N_{\gamma}}{d \phi d \phi^{\prime}}
$$

We define $\sigma=\phi+\phi^{\prime}$ and $\tau=\left(\phi^{\prime}-\phi\right) / 2$, respectively, double the average of, and half the time between, emissions at different points on the electron trajectory. In a circularly polarized monochromatic plane wave, the integrand above is independent of $\sigma$ and therefore (as already discussed) infinite. However, the rate $W=N_{\gamma} / \int d \sigma$ is finite, which gives

$$
W=\int d \tau \frac{d W}{d \tau} .
$$

In order to compare with our numerical results we require the differential rate with respect to a kinematic variable, and for this we choose the low-energy (classical) limit of the variable (3), which is

$$
s=\frac{k \cdot k^{\prime}}{k \cdot p}=\frac{\chi_{\gamma}}{\chi_{e}},
$$

as can be checked by reintroducing $\hbar$ and expanding in powers of the photon momentum. The differential rate (summed over all harmonics) we consider is

$$
\begin{aligned}
\frac{\partial W}{\partial s}= & -\frac{\alpha}{2 \pi b_{0}} \int_{-\infty}^{\infty} \frac{d \tau}{\tau}\left(\sin \frac{\tau s}{b_{0}}-\sin \frac{\tau s \mu}{b_{0}}\right. \\
& \left.-2 \tau^{2} a_{0}^{2} \operatorname{sinc}^{2} \tau \sin \frac{\tau s \mu}{b_{0}}\right),
\end{aligned}
$$

in which $\tau$ is as above, $b_{0}=k \cdot p / m^{2}$ and $\mu$ is the ratio of Kibble's mass $M^{2}$ to $m^{2}$ [67] in a monochromatic wave being equal to

$$
M^{2}(\tau) / m^{2}=\mu(\tau)=1+a_{0}^{2}\left(1-\operatorname{sinc}^{2} \tau\right) .
$$

Note that the asymptotic limit of $M^{2}$ is the effective mass squared, $m_{*}^{2}$, introduced above. Since the integrand in Eq. (15) 
is even in $\tau$, we are dealing with integrals of the form

$$
\int_{0}^{\infty} d \tau f(\tau) e^{i \Phi(\tau)}
$$

where the phase

$$
\Phi(\tau)=\frac{s \tau}{b_{0}}\left[1+a_{0}^{2}\left(1-\operatorname{sinc}^{2} \tau\right)\right]
$$

has no extrema on the real line. The LCF approximation corresponds to expanding $\Phi(t)$ to next-to-lowest order in $\tau$, giving

$$
\Phi(\tau) \simeq \Phi_{\mathrm{LCF}}(\tau)=\frac{s \tau}{b_{0}}\left[1+\frac{a_{0}^{2} \tau^{2}}{3}\right] .
$$

Since the condition for neglecting the next term in this expansion is $\tau^{2} \ll 15 / 2$, we see that using the expansion in Eq. (19) assumes that only a finite range $t \in\left[0, \tau_{\max }\right]$ contributes to photon emission and that the total integral can be approximated as

$$
\int_{0}^{\infty} d \tau f(\tau) e^{i \Phi(\tau)} \approx \int_{0}^{\tau_{\max }} d \tau f(\tau) e^{i \Phi_{\mathrm{LCF}}(\tau)} .
$$

Recall that $\tau$ measures the correlation of radiation emitted from different points on the electron trajectory. If only small $\tau$ is included, then emission is assumed to be "local." Writing $x^{-}=t-z$, we note that $\tau=k \cdot\left(x^{\prime}-x\right) / 2=\pi\left(x^{-}-x^{-}\right) / \lambda$ for the external field wavelength $\lambda$ and observe that the small- $\tau$ limit can be understood as the large- $\lambda$ limit, thereby demonstrating the equivalence of the LCF approximation and the constant crossed field limit in the current problem. When the LCF approximation is applied to Eq. (15), we recover the constant crossed field expression upon performing the $\tau$ integral,

$$
\frac{\partial W_{\mathrm{LCF}}}{\partial s}=-\frac{\alpha}{b_{0}}\left(\mathrm{Ai}_{1}(z)+\frac{2}{z} \operatorname{Ai}^{\prime}(z)\right)
$$

where $z=\left(s / a_{0} b_{0}\right)^{2 / 3}$. Now, if the LCF assumptions approximate the original integral well, the majority of the full integral in $t$ must be included in the assumption (20), which implies $s \tau_{\max } / b_{0} \gtrsim 1$. If $\tau_{\max } \sim O(1)$, this condition can be fulfilled for $s / b_{0} \gg 1$. However, $s$ is not always confined to this range, as is most evident for the first harmonic for which, from Eq. (4), $s / b_{0} \leqslant 1 /\left(1+a_{0}^{2}\right)<1$. Therefore the LCF assumptions break down here; this is demonstrated in Fig. 10, where the LCF approximation misses the first harmonic structure, precisely as is seen in the numerical simulation in Fig. 4.

In fact we can show explicitly that information pertaining to the first harmonic is contained in the large $\tau$ (i.e., largedistance) expansion of the integrand, in particular in the asymptotic expansion of the effective mass. To do so we simply replace $M^{2}$ with its asymptotic limit $m_{*}^{2}$ in Eq. (15) and evaluate [68, §3.828-3]

$$
\frac{\partial W}{\partial s} \rightarrow \frac{\alpha a_{0}^{2}}{\pi b_{0}} \int \frac{d \tau}{\tau} \sin ^{2} \tau \sin \frac{2 s \tau}{y_{1}}=\frac{\alpha a_{0}^{2}}{2 b_{0}} \theta\left(y_{1}-s\right),
$$

(where $\theta(\cdot)$ is the Heaviside function with $\theta(0)=1 / 2$ ) which is precisely the range of the first harmonic and also gives the jump discontinuity clearly visible in the emission spectrum, marked in Fig. 10. The LCF approximation knows nothing about the large-distance expansion of the effective mass and is

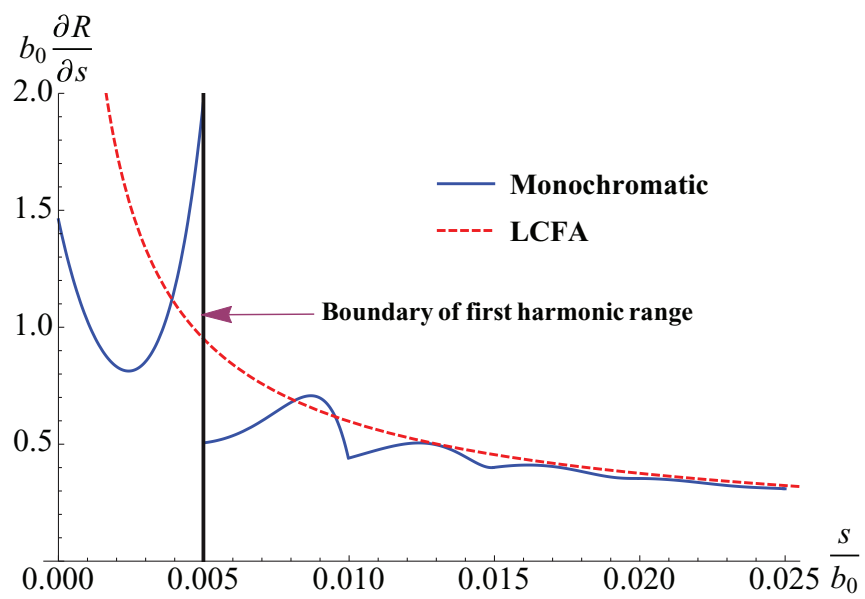

FIG. 10. (Color online) The full spectrum for a monochromatic field (blue solid line) and the LCF approximation to it (red dashed line, marked 'LCFA'). $a_{0}=20$ to illustrate. The vertical black line marks the boundary of the first harmonic.

blind to harmonic structure, in particular, the first harmonic; the LCF approximation to the emission spectrum, used either analytically as in Fig. 10 or numerically as in Fig. 4, goes smoothly through the jump at the edge of the first harmonic range.

\section{CONCLUSIONS}

We have tested current numerical implementations of strong-field electrodynamics by comparing their predicted spectra with known analytical results. Focusing on photon emission from an electron, we have found that common PIC-based models correctly reproduce many features of the emission spectra. The high-energy tails of the distributions are well matched, as are the angular distributions, especially in the highly relativistic limit where the electrons emit almost entirely forward, matching the assumption that goes into the codes.

What the numerical model fails to reproduce is the harmonic structure of the photon distribution in frequency space. The well-known peaks and troughs associated with (higher) harmonic generation, which are the distinct, indeed measured, signals of intensity and multiphoton effects, are missed. It has been shown that this discrepancy can be attributed to the use of the locally constant field approximation, which is the standard way of including strong-field QED processes in numerical models.

Despite the good overall agreement between theory and numerics, our investigation prompts further enquiry. By extending our numerical and analytical results to the quantum regime, other processes such as pair production can also be scrutinized.

\section{ACKNOWLEDGMENTS}

C.H. thanks Dermot Green and Adam Noble for useful discussions. C.H. is supported by EPSRC, Grant No. EP/I029206/1-YOTTA, and A.I. is supported by The Swedish Research Council, Contract No. 2011-4221. 
[1] M. Marklund and P. K. Shukla, Rev. Mod. Phys. 78, 591 (2006).

[2] G. V. Dunne, Eur. Phys. J. D 55, 327 (2009).

[3] F. Ehlotzky, K. Krajewska, and J. Z. Kaminski, Rep. Prog. Phys. 72, 046401 (2009).

[4] T. Heinzl, Int. J. Mod. Phys. A 27, 1260010 (2012).

[5] J. Redondo and A. Ringwald, Contemp. Phys. 52, 211 (2011).

[6] J. Jaeckel and A. Ringwald, Annu. Rev. Nucl. Part. Sci. 60, 405 (2010).

[7] A. Di Piazza, C. Müller, K. Z. Hatsagortsyan, and C. H. Keitel, Rev. Mod. Phys. 84, 1177 (2012).

[8] A. Nikishov and V. Ritus, Sov. Phys. JETP 19, 529 (1964).

[9] A. Nikishov and V. Ritus, Sov. Phys. JETP 19, 1191 (1964).

[10] ELMIS-A fully parallel fourier-based multi-dimensional pic code for laser-plasma interaction simulations, http://icnsp2011.pppl.gov/abstracts/Korzhimanov_A_ELMIS a fully parallel.pdf.

[11] For OSIRIS, see https://plasmasim.physics.ucla.edu/codes/osiris.

[12] Project plasma simulation code, http://www.plasma-simulationcode.net.

[13] A. R. Bell and J. G. Kirk, Phys. Rev. Lett. 101, 200403 (2008).

[14] I. V. Sokolov, N. M. Naumova, J. A. Nees, and G. A. Mourou, Phys. Rev. Lett. 105, 195005 (2010).

[15] E. N. Nerush, I. Y. Kostyukov, A. M. Fedotov, N. B. Narozhny, N. V. Elkina, and H. Ruhl, Phys. Rev. Lett. 106, 035001 (2011).

[16] T. G. Blackburn, C. P. Ridgers, J. G. Kirk, and A. R. Bell, Phys. Rev. Lett. 112, 015001 (2014).

[17] A. A. Mironov, N. B. Narozhny, and A. M. Fedotov, Phys. Lett. A 378, 3254 (2014).

[18] N. V. Elkina, A. M. Fedotov, I. Y. Kostyukov, M. V. Legkov, N. B. Narozhny, E. N. Nerush, and H. Ruhl, Phys. Rev. Spec. Top.-Accel. Beams 14, 054401 (2011).

[19] C. Ridgers, J. Kirk, R. Duclous, T. Blackburn, C. Brady, K. Bennett, T. Arber, and A. Bell, J. Comput. Phys. 260, 273 (2014).

[20] The Vulcan 10 Petawatt Project, http://www.clf.stfc.ac.uk/ New+Initiatives/The+Vulcan+10+Petawatt+Project/14684.aspx.

[21] The Extreme Light Infrastructure (eli) Project, http://www.elilaser.eu/index.html.

[22] Exawatt Center for Extreme Light Studies (xcels), http://www.xcels.iapras.ru/.

[23] V. Yanovsky, V. Chvykov, G. Kalinchenko, P. Rousseau, T. Planchon, T. Matsuoka, A. Maksimchuk, J. Nees, G. Cheriaux, G. Mourou, and K. Krushelnick, Opt. Express 16, 2109 (2008).

[24] B. King, N. Elkina, and H. Ruhl, Phys. Rev. A 87, 042117 (2013).

[25] A. I. Nikishov and V. I. Ritus, Sov. Phys. JETP 25, 1135 (1967).

[26] T. Erber, Rev. Mod. Phys. 38, 626 (1966).

[27] V. Baier and V. Katkov, Phys. Lett. A 374, 2201 (2010).

[28] T. Heinzl, A. Ilderton, and M. Marklund, Phys. Lett. B 692, 250 (2010).

[29] T. Nousch, D. Seipt, B. Kampfer, and A. Titov, Phys. Lett. B 715, 246 (2012).

[30] V. Ritus, Nucl. Phys. B 44, 236 (1972).

[31] V. N. Baier, V. M. Katkov, and V. M. Strakhovenko, Sov. Phys. Nucl. Phys. 14, 572 (1972).

[32] H. Hu, C. Muller, and C. H. Keitel, Phys. Rev. Lett. 105, 080401 (2010).
[33] A. Ilderton, Phys. Rev. Lett. 106, 020404 (2011).

[34] B. King and H. Ruhl, Phys. Rev. D 88, 013005 (2013).

[35] F. Sauter, Z. Phys. 69, 742 (1931).

[36] W. Heisenberg and H. Euler, Z. Phys. 98, 714 (1936).

[37] J. Schwinger, Phys. Rev. 82, 664 (1951).

[38] V. Ritus, J. Sov. Laser Res. 6, 497 (1985).

[39] B. King, arXiv:1410.5478.

[40] C. Harvey, T. Heinzl, and M. Marklund, Phys. Rev. D 84, 116005 (2011).

[41] C. Harvey, T. Heinzl, and A. Ilderton, Phys. Rev. A 79, 063407 (2009).

[42] M. Boca and V. Florescu, Phys. Rev. A 80, 053403 (2009).

[43] T. Heinzl, D. Seipt, and B. Kampfer, Phys. Rev. A 81, 022125 (2010).

[44] F. Mackenroth and A. Di Piazza, Phys. Rev. A 83, 032106 (2011).

[45] D. M. Volkov, Z. Phys. 94, 250 (1935).

[46] N. Sengupta, Bull. Calcutta Math. Soc. 41, 187 (1949).

[47] C. Harvey, T. Heinzl, A. Ilderton, and M. Marklund, Phys. Rev. Lett. 109, 100402 (2012).

[48] T. J. Englert and E. A. Rinehart, Phys. Rev. A 28, 1539 (1983).

[49] S.-Y. Chen, A. Maksimchuk, and D. Umstadter, Nature (London) 396, 653 (1998).

[50] M. Iinuma, K. Matsukado, I. Endo, M. Hashida, K. Hayashi, A. Kohara, F. Matsumoto, Y. Nakanishi, S. Sakabe, S. Shimizu, T. Tauchi, K. Yamamoto, and T. Takahashi, Phys. Lett. A 346, 255 (2005).

[51] A. Ilderton and G. Torgrimsson, Phys. Rev. D 87, 085040 (2013).

[52] R. Duclous, J. G. Kirk, and A. R. Bell, Plasma Phys. Controlled Fusion 53, 015009 (2011).

[53] J. D. Jackson, Classical Electrodynamics, 3rd ed. (Wiley, New York, 1999).

[54] D. Seipt and B. Kampfer, Phys. Rev. D 85, 101701 (2012).

[55] F. Mackenroth and A. Di Piazza, Phys. Rev. Lett. 110, 070402 (2013).

[56] R. J. Glauber, Phys. Rev. 84, 395 (1951).

[57] D. Yennie, S. C. Frautschi, and H. Suura, Ann. Phys. 13, 379 (1961).

[58] K. E. Eriksson, Il Nuovo Cimento Ser. 10, 19 (1961).

[59] R. Horan, M. Lavelle, and D. McMullan, J. Math. Phys. 41, 4437 (2000).

[60] M. E. Peskin and D. V. Schroeder, An Introduction to Quantum Field Theory (Addison-Wesley, Reading, MA, 1995).

[61] A. Di Piazza, K. Z. Hatsagortsyan, and C. H. Keitel, Phys. Rev. Lett. 105, 220403 (2010).

[62] V. Dinu, T. Heinzl, and A. Ilderton, Phys. Rev. D 86, 085037 (2012).

[63] V. Dinu, Phys. Rev. A 87, 052101 (2013).

[64] D. G. Green and C. N. Harvey, Phys. Rev. Lett. 112, 164801 (2014).

[65] D. G. Green and C. N. Harvey, arXiv:1410.4055.

[66] K. Krajewska, M. Twardy, and J. Z. Kaminski, Phys. Rev. A 89, 052123 (2014).

[67] T. Kibble, A. Salam, and J. Strathdee, Nucl. Phys. B 96, 255 (1975).

[68] I. Gradshteyn and I. Ryzhik, Table of Integrals, Series, and Products (Academic Press, San Diego, 2007). 